\title{
On the behaviour of Poynting vector in material media with weak optical activity
}

\author{
Adamenko D., Kostyrko M. and Vlokh R. \\ Institute of Physical Optics, 23 Dragomanov St., 79005 Lviv, Ukraine, \\ e-mail: vlokh@ifo.lviv.ua
}

Received: 04.07.2011

\begin{abstract}
We have obtained phenomenological relation for the Poynting vector of electromagnetic wave propagating in crystals that possess a so-called weak optical activity. The appearance of transverse component of the Poynting vector and a transverse shift of the optical beam due to spin-orbit interaction are discussed.
\end{abstract}

Keywords: Poynting vector, weak optical activity

PACS: $78.20 . \mathrm{Ek}$

UDC: 535.56

\section{Introduction}

It is well known that optical activity is described by accounting for inhomogeneity of electric induction $D_{j}$ appearing in the relation for electric field $E_{i}$ of an optical wave that propagates through a medium:

$$
E_{i}=B_{i j}^{0} D_{j}+\gamma_{i j k} \frac{\partial D_{j}}{\partial x_{k}},
$$

where $B_{i j}^{0}$ denotes the optical-frequency impermeability tensor, $\gamma_{i j k}$ the third-rank antisymmetric polar tensor $\left(\gamma_{i j k}=-\gamma_{j i k}\right)$, and $x_{k}$ the coordinate. Using the known duality relation,

$$
\frac{2 \pi}{\lambda} \gamma_{i j k}=\delta_{i j l} g_{l k}
$$

one can reduce the tensor $\gamma_{i j k}$ to a (generally nonsymmetric) second-rank axial gyration tensor $g_{l k}$ (with $\delta_{i j l}$ being the unit antisymmetric Levi-Civita tensor). Then Eq. (1) may be rewritten as

$$
E_{i}=\left(B_{i j}^{0}+i \delta_{i j l} g_{l k} m_{k}\right) D_{j}
$$

where $k_{k}=\frac{2 \pi}{\lambda} m_{k}$ denotes the wave vector of light and $m_{k}$ the unit vector parallel to $k_{k}$. In its turn, the nonsymmetric gyration tensor can be decomposed into symmetric and antisymmetric parts:

$$
g_{l k}=g_{l k}^{s}+g_{l k}^{a s},
$$

A common point of view is that the optical activity effect is purely associated with the symmetric part of the gyration tensor. Scarce experimental data is available on manifestations of a kind of optical activity concerned with the antisymmetric part of the gyration tensor which is dual to some polar vector $h_{r}$, 


$$
g_{l k}^{a s}=\delta_{l k r} h_{r},
$$

while the results of relevant theoretical analysis are very poor [1-5].

Recently, we have shown that this effect known as a weak optical activity should manifest itself in some changes of refractive indices and optical birefringence [6]. Moreover, if both the common optical activity and the weak one are simultaneously present, the polarisation state of one of the eigenwaves in crystal acquires a complicated longitudinally-transverse elliptical polarisation [7]. This novel polarisation eigenstate comprises two elliptical states, one of which is transversely elliptical and the other longitudinally elliptical.

Due to the Neumann's symmetry principle, the point symmetry groups for which $h_{r} \neq 0$ are 1, 2, $m, m m 2,3,3 m, 4,4 m m, 6,6 m m, \infty$, and $\infty m m$. Among these groups, only four reveal no common optical activity $\left(g_{l k}^{s}=0\right): 3 m, 4 m m, 6 m m$, and $\infty \mathrm{mm}$. Eq. (3) for these media may be written as

$$
E_{i}=\left(B_{i j}^{0}+i \delta_{i j l} \delta_{l k r} h_{r} m_{k}\right) D_{j} .
$$

It is obvious that only a single component of the $h_{r}$ vector remains nonzero for the crystals that belong to the point groups $3 m, 4 m m, 6 m m$ (namely, we have $h_{3} \neq 0$ ).

\section{Results of analysis}

Let a plane electromagnetic wave propagate through a transparent, anisotropic, magnetically nonordered, though spatially dispersive, medium. Consider the Poynting vector represented in the usual form $[8,9]$

$$
S_{k}^{0}+S_{k}^{1}=u_{k} W
$$

where $u_{k} W$ is the total electromagnetic energy flow, $W$ the energy density, $S_{k}^{0}$ the energy flow without accounting for the spatial dispersion effect, $S_{k}^{1}$ the extra energy flow that appears if the spatial dispersion is present, $u_{k}=\partial \omega / \partial k_{k}$ means the group velocity, and $\omega$ the frequency of wave. The relations for the energy density and the time-averaged Poynting vector components are given by

$$
\begin{aligned}
& W=\frac{1}{4}\left\{\varepsilon_{0} \frac{\partial\left(\omega \varepsilon_{i j}\right)}{\partial \omega} E_{0 j} E_{0 i}^{*}+\frac{1}{\mu_{0}} B_{0 i} B_{0 i}^{*}\right\}, \\
& S_{k}^{0}=\frac{1}{4 \mu_{0}}\left\{\left[E_{0 j} \times B_{0 i}^{*}\right]+\left[E_{0 i}^{*} \times B_{0 j}\right]\right\}, \\
& S_{k}^{1}=-\left.\frac{1}{4} \varepsilon_{0} \omega \frac{\partial \varepsilon_{i j}}{\partial k_{k}}\right|_{\omega=\mathrm{const}} E_{0 j} E_{0 i}^{*},
\end{aligned}
$$

where $\varepsilon_{i j}$ stands for the dielectric permittivity, $\varepsilon_{0}$ the dielectric permittivity of vacuum, $E_{0 j}, E_{0 i}^{*}$ the amplitudes of electric field components, $B_{0}, B_{0}^{*}$ the amplitudes of the magnetic induction, and $\mu_{0}$ the magnetic constant. When specifying Eq. (10), one needs equations for the dielectric permittivity tensor components and their wave-vector derivatives for the media that possess the weak optical activity. 
Let us consider a particular case of optically uniaxial crystals, for which the following conditions are satisfied:

$$
h_{3} \neq 0 ; h_{1}=h_{2}=0 ; B_{11}^{0}=B_{22}^{0} \neq B_{33}^{0} ; m_{2}=m_{3}=0 ; m_{1}=1 .
$$

The matrix that couples the components of the electric field and electric displacement vectors becomes

so that we get a system of equations

\begin{tabular}{c|ccc} 
& $D_{1}$ & $D_{2}$ & $D_{3}$ \\
\hline$E_{1}$ & $B_{11}^{0}$ & 0 & $i h_{3} m_{1}$ \\
$E_{2}$ & 0 & $B_{11}^{0}$ & 0 \\
$E_{3}$ & $-i h_{3} m_{1}$ & 0 & $B_{33}^{0}$
\end{tabular},

$$
\left\{\begin{array}{l}
E_{1}=B_{11}^{0} D_{1}+i h_{3} m_{1} D_{3} \\
E_{2}=B_{11}^{0} D_{2} \\
E_{3}=-i h_{3} m_{1} D_{1}+B_{33}^{0} D_{3}
\end{array} .\right.
$$

The corresponding relations that describe the electric induction of electromagnetic wave as a function of its electric field may be found as

$$
\left\{\begin{array}{l}
D_{1}=\frac{B_{33}^{0}}{B_{11}^{0} B_{33}^{0}-\left(h_{3} m_{1}\right)^{2}} E_{1}-i \frac{h_{3} m_{1}}{B_{11}^{0} B_{33}^{0}-\left(h_{3} m_{1}\right)^{2}} E_{3} \\
D_{2}=\frac{1}{B_{11}^{0}} E_{2} \\
D_{3}=i \frac{h_{3} m_{1}}{B_{11}^{0} B_{33}^{0}-\left(h_{3} m_{1}\right)^{2}} E_{1}+\left(\frac{1}{B_{33}^{0}}+\frac{\left(h_{3} m_{1}\right)^{2}}{B_{33}^{0}\left(B_{11}^{0} B_{33}^{0}-\left(h_{3} m_{1}\right)^{2}\right)}\right) E_{3}
\end{array} .\right.
$$

Thus, the dielectric permittivity tensor is as follows:

$$
\varepsilon_{i j}=\left|\begin{array}{ccc}
\frac{B_{33}^{0}}{B_{11}^{0} B_{33}^{0}-\left(h_{3} m_{1}\right)^{2}} & 0 & -\frac{i h_{3} m_{1}}{B_{11}^{0} B_{33}^{0}-\left(h_{3} m_{1}\right)^{2}} \\
0 & \frac{1}{B_{11}^{0}} & 0 \\
\frac{i h_{3} m_{1}}{B_{11}^{0} B_{33}^{0}-\left(h_{3} m_{1}\right)^{2}} & 0 & \frac{1}{B_{33}^{0}}+\frac{\left(h_{3} m_{1}\right)^{2}}{B_{33}^{0}\left(B_{11}^{0} B_{33}^{0}-\left(h_{3} m_{1}\right)^{2}\right)}
\end{array}\right| .
$$

Taking into account the relation $h_{3} m_{1}=h_{3} k_{1} \lambda / 2 \pi=h_{3} k_{1} c / \omega$, one can rewrite the matrix given by Eq. (15) as

$$
\varepsilon_{i j}=\left|\begin{array}{ccc}
\frac{\omega^{2} B_{33}^{0}}{\omega^{2} B_{11}^{0} B_{33}^{0}-\left(h_{3} k_{1} c\right)^{2}} & 0 & -i \frac{\omega h_{3} k_{1} c}{\omega^{2} B_{11}^{0} B_{33}^{0}-\left(h_{3} k_{1} c\right)^{2}} \\
0 & \frac{1}{B_{11}^{0}} & 0 \\
i \frac{\omega h_{3} k_{1} c}{\omega^{2} B_{11}^{0} B_{33}^{0}-\left(h_{3} k_{1} c\right)^{2}} & 0 & \frac{1}{B_{33}^{0}}\left(1+\frac{\left(h_{3} k_{1} c\right)^{2}}{\omega^{2} B_{11}^{0} B_{33}^{0}-\left(h_{3} k_{1} c\right)^{2}}\right)
\end{array}\right| .
$$


Then the wave-vector derivatives of the dielectric tensor components reduce to

$$
\begin{aligned}
& \left.\frac{\partial\left(\varepsilon_{11}\right)}{\partial k_{1}}\right|_{\omega=\mathrm{const}}=\frac{2 k_{1} \omega^{2} B_{33}^{0}\left(h_{3} c\right)^{2}}{\left[\omega^{2} B_{11}^{0} B_{33}^{0}-\left(h_{3} k_{1} c\right)^{2}\right]^{2}}, \\
& \left.\frac{\partial\left(\varepsilon_{22}\right)}{\partial k_{1}}\right|_{\omega=\mathrm{const}}=0, \\
& \left.\frac{\partial\left(\varepsilon_{33}\right)}{\partial k_{1}}\right|_{\omega=\mathrm{const}}=\frac{2 k_{1} \omega^{2}\left(h_{3} c\right)^{2} B_{11}^{0}}{\left[\omega^{2} B_{11}^{0} B_{33}^{0}-\left(h_{3} k_{1} c\right)^{2}\right]^{2}}, \\
& \left.\frac{\partial\left(\varepsilon_{13}\right)}{\partial k_{1}}\right|_{\omega=\mathrm{const}}=-i \omega h_{3} c \frac{\omega^{2} B_{11}^{0} B_{33}^{0}+\left(h_{3} k_{1} c\right)^{2}}{\left[\omega^{2} B_{11}^{0} B_{33}^{0}-\left(h_{3} k_{1} c\right)^{2}\right]^{2}}
\end{aligned}
$$

Using Eqs. (17)-(20), one can represent Eq. (10) as

$$
\begin{aligned}
& S_{1}^{1}=-\frac{1}{2} \varepsilon_{0} \frac{k_{1} \omega^{3}\left(h_{3} c\right)^{2}}{\left[\omega^{2} B_{11}^{0} B_{33}^{0}-\left(h_{3} k_{1} c\right)^{2}\right]^{2}}\left(B_{33}^{0} E_{01} E_{01}^{*}+B_{11}^{0} E_{03} E_{03}^{*}\right) \\
& +\frac{1}{4} i \varepsilon_{0} \omega^{2} h_{3} c \frac{\omega^{2} B_{11}^{0} B_{33}^{0}+\left(h_{3} k_{1} c\right)^{2}}{\left[\omega^{2} B_{11}^{0} B_{33}^{0}-\left(h_{3} k_{1} c\right)^{2}\right]^{2}}\left(E_{03} E_{01}^{*}-E_{01} E_{03}^{*}\right)
\end{aligned} .
$$

In the assumptions that $E_{01}^{*}$ and $E_{03}^{*}$ are real, Eq. (21) for the component $S_{1}^{1}$ of the Poynting vector consists only of real part. The real part appearing in Eq. (21) is associated with some changes in the group velocity of electromagnetic wave caused by the weak optical activity.

As follows from Eqs. (14), the component $D_{1}$ is a pure result of the weak optical activity. Due to the condition $D_{1} \perp B_{2} \perp k_{3}$, the component $k_{3}$ of the wave vector has to arise, too. This means that Eqs. (17)-(20) should also include the derivatives of $k_{3}$, thus inevitably leading to appearance of the component $S_{3}^{1}$ of the Poynting vector. In fact this implies that the light beam should 'drift' along $Z$ direction while propagating in crystals with the weak optical activity. This effect can be easily explained when taking into account that the elliptically polarised photon, with its polarisation ellipse belonging to $X Z$ plane, should possess a spin-orbit momentum component $s_{2}$. The existence of this component of spin angular momentum would lead to the drift of photon mentioned above, as a result of a spin-orbit interaction and an optical Magnus effect [10-12].

Now let us derive the relation for the $S_{3}^{1}$ component of the Poynting vector. As already mentioned, the weak optical activity leads to inclination of the $\vec{D}$ component of electric induction of the electromagnetic wave propagating along $X$ direction, with appearance of $D_{1}$ component. The ratio of these components can be expressed as

$$
\frac{D_{1}}{D_{3}}=\frac{B_{33}^{0} E_{1}-i h_{3} m_{1} E_{3}}{i h_{3} m_{1} E_{1}+B_{11}^{0} E_{3}}=-\frac{k_{3}}{k_{1}} .
$$

Taking into account that $m_{1}=k_{1} c / \omega$, one can rewrite Eq. (22) in the form of quadratic equation with respect to the wave vector $k_{1}$ :

$$
i h_{3}\left(k_{1}\right)^{2} c E_{3}-k_{1} E_{1}\left(\omega B_{33}^{0}+i h_{3} k_{3} c\right)-\omega B_{11}^{0} k_{3} E_{3}=0
$$


with the solutions

$$
\begin{aligned}
& \left(k_{1}\right)_{1,2}=\frac{1}{2 i h_{3} c E_{3}} \\
& \times\left[\omega B_{33}^{0} E_{1}+i h_{3} k_{3} c E_{1} \pm \sqrt{\omega^{2}\left(B_{33}^{0}\right)^{2} E_{1}^{2}-\left(k_{3}\right)^{2}\left(h_{3} c\right)^{2} E_{1}^{2}+2 i h_{3} c \omega k_{3}\left(B_{33}^{0} E_{1}^{2}+2 B_{11}^{0} E_{3}^{2}\right)}\right]
\end{aligned}
$$

of which derivatives can be represented as

$$
\left.\frac{\partial k_{1}}{\partial k_{3}}\right|_{\omega=\mathrm{const}}=\frac{E_{1}}{2 E_{3}} \pm \frac{\omega\left(B_{33}^{0} E_{1}^{2}+2 B_{11}^{0} E_{3}^{2}\right)+i k_{3} h_{3} c E_{1}^{2}}{2 E_{3} \sqrt{\omega^{2}\left(B_{33}^{0}\right)^{2} E_{1}^{2}-\left(k_{3}\right)^{2}\left(h_{3} c\right)^{2} E_{1}^{2}+2 i h_{3} c \omega k_{3}\left(B_{33}^{0} E_{1}^{2}+2 B_{11}^{0} E_{3}^{2}\right)}} .
$$

Considering Eq. (22), one can rewrite Eq. (25) as

$$
\left.\frac{\partial k_{1}}{\partial k_{3}}\right|_{\omega=\text { const }}=\frac{E_{1}}{2 E_{3}} \pm \frac{\left(\omega^{2} B_{11}^{0} B_{33}^{0}-\left(h_{3} k_{1} c\right)^{2}\right) E_{1}^{2}+2 i h_{3} k_{1} c \omega B_{11}^{0} E_{1} E_{3}+2\left(\omega B_{11}^{0}\right)^{2} E_{3}^{2}}{2\left(\omega^{2} B_{11}^{0} B_{33}^{0}+\left(h_{3} k_{1} c\right)^{2}\right) E_{1} E_{3}-4 i h_{3} k_{1} c \omega B_{11}^{0} E_{3}^{2}} .
$$

Since the $S_{3}^{1}$ vector is defined by the relation

$$
S_{3}^{1}=-\left.\frac{1}{4} \varepsilon_{0} \omega \frac{\partial \varepsilon_{i j}^{\prime}}{\partial k_{3}}\right|_{\omega=\text { const }} E_{0 j} E_{0 i}^{*}=-\left.\left.\frac{1}{4} \varepsilon_{0} \omega \frac{\partial \varepsilon_{i j}^{\prime}}{\partial k_{1}}\right|_{\omega=\text { const }} \frac{\partial k_{1}}{\partial k_{3}}\right|_{\omega=\text { const }} E_{0 j} E_{0 i}^{*}=\left.\frac{\partial k_{1}}{\partial k_{3}}\right|_{\omega=\text { const }} S_{1}^{1},
$$

one can write out the final expression for the $Z$ component of the Poynting vector:

$$
\begin{aligned}
& S_{3}^{1}=-\frac{\varepsilon_{0}\left(h_{3} c\right)^{2} \omega^{3} k_{1}\left(B_{33}^{0} E_{01} E_{01}^{*}+B_{11}^{0} E_{03} E_{03}^{*}\right)}{4\left[\omega^{2} B_{11}^{0} B_{33}^{0}-\left(h_{3} k_{1} c\right)^{2}\right]^{2}} \\
& \times\left[\frac{E_{1}}{E_{3}} \pm \frac{\left(\left(\omega^{2} B_{11}^{0} B_{33}^{0}\right)^{2}-\left(h_{3} k_{1} c\right)^{4}\right) E_{1}^{3} E_{3}+2\left(\omega B_{11}^{0}\right)^{2}\left(\omega^{2} B_{11}^{0} B_{33}^{0}-\left(h_{3} k_{1} c\right)^{2}\right) E_{1} E_{3}^{3}}{\left(\omega^{2} B_{11}^{0} B_{33}^{0}+\left(h_{3} k_{1} c\right)^{2}\right)^{2} E_{1}^{2} E_{3}^{2}+4\left(h_{3} k_{1} c \omega B_{11}^{0}\right)^{2} E_{3}^{4}}\right] \\
& -\frac{\varepsilon_{0}\left(h_{3} c\right)^{2} \omega^{3} k_{1}\left(B_{33}^{0} E_{01} E_{01}^{*}+B_{11}^{0} E_{03} E_{03}^{*}\right)}{4\left[\omega^{2} B_{11}^{0} B_{33}^{0}-\left(h_{3} k_{1} c\right)^{2}\right]^{2}} \times \frac{4 i h_{3} k_{1} c \omega^{3}\left(B_{11}^{0}\right)^{2}\left[B_{33}^{0} E_{1}^{2} E_{3}^{2}+B_{11}^{0} E_{3}^{4}\right]}{\left(\omega^{2} B_{11}^{0} B_{33}^{0}+\left(h_{3} k_{1} c\right)^{2}\right)^{2} E_{1}^{2} E_{3}^{2}+4\left(h_{3} k_{1} c \omega B_{11}^{0}\right)^{2} E_{3}^{4}} .
\end{aligned}
$$

In the assumptions $E_{01}=E_{01}^{*}=E_{1}$ and $E_{03}=E_{03}^{*}=E_{3}$, Eq. (28) reads as

$$
\begin{aligned}
& S_{3}^{1}=S_{1}^{1} \times\left.\frac{\partial k_{1}}{\partial k_{3}}\right|_{\omega=\text { const }}==-\frac{\varepsilon_{0}\left(h_{3}\right)^{2} k_{1} c^{2} \omega^{3}\left(B_{33}^{0} E_{1}^{2}+B_{11}^{0} E_{3}^{2}\right)}{4\left[\omega^{2} B_{11}^{0} B_{33}^{0}-\left(h_{3} k_{1} c\right)^{2}\right]^{2}} \times \\
& {\left[\frac{E_{1}}{E_{3}} \pm \frac{\left(\left(\omega^{2} B_{11}^{0} B_{33}^{0}\right)^{2}-\left(h_{3} k_{1} c\right)^{4}\right) E_{1}^{3} E_{3}+2\left(\omega B_{11}^{0}\right)^{2}\left(\omega^{2} B_{11}^{0} B_{33}^{0}-\left(h_{3} k_{1} c\right)^{2}\right) E_{1} E_{3}^{3}}{\left(\omega^{2} B_{11}^{0} B_{33}^{0}+\left(h_{3} k_{1} c\right)^{2}\right)^{2} E_{1}^{2} E_{3}^{2}+4\left(h_{3} k_{1} c \omega B_{11}^{0}\right)^{2} E_{3}^{4}}\right]} \\
& -\frac{\varepsilon_{0}\left(h_{3}\right)^{2} k_{1} c^{2} \omega^{3}\left(B_{33}^{0} E_{1}^{2}+B_{11}^{0} E_{3}^{2}\right)}{4\left[\omega^{2} B_{11}^{0} B_{33}^{0}-\left(h_{3} k_{1} c\right)^{2}\right]^{2}} \times \frac{4 i h_{3} k_{1} c \omega^{3}\left(B_{11}^{0}\right)^{2}\left[B_{33}^{0} E_{1}^{2} E_{3}^{2}+B_{11}^{0} E_{3}^{4}\right]}{\left(\omega^{2} B_{11}^{0} B_{33}^{0}+\left(h_{3} k_{1} c\right)^{2}\right)^{2} E_{1}^{2} E_{3}^{2}+4\left(h_{3} k_{1} c \omega B_{11}^{0}\right)^{2} E_{3}^{4}} .
\end{aligned}
$$

Now one can extract the real part of the $Z$ component of the Poynting vector basing on Eq. (29). It is equal to 


$$
\begin{aligned}
& \left(S_{3}^{1}\right)_{\operatorname{Re}}=-\frac{\varepsilon_{0}\left(h_{3}\right)^{2} k_{1} c^{2} \omega^{3}\left(B_{33}^{0} E_{1}^{2}+B_{11}^{0} E_{3}^{2}\right)}{4\left[\omega^{2} B_{11}^{0} B_{33}^{0}-\left(h_{3} k_{1} c\right)^{2}\right]^{2}} \\
& \times\left[\frac{E_{1}}{E_{3}} \pm \frac{\left(\left(\omega^{2} B_{11}^{0} B_{33}^{0}\right)^{2}-\left(h_{3} k_{1} c\right)^{4}\right) E_{1}^{3} E_{3}+2\left(\omega B_{11}^{0}\right)^{2}\left(\omega^{2} B_{11}^{0} B_{33}^{0}-\left(h_{3} k_{1} c\right)^{2}\right) E_{1} E_{3}^{3}}{\left(\omega^{2} B_{11}^{0} B_{33}^{0}+\left(h_{3} k_{1} c\right)^{2}\right)^{2} E_{1}^{2} E_{3}^{2}+4\left(h_{3} k_{1} c \omega B_{11}^{0}\right)^{2} E_{3}^{4}}\right],
\end{aligned}
$$

or

$$
\begin{aligned}
& \left(S_{3}^{1}\right)_{\operatorname{Re}}=-\frac{\varepsilon_{0}\left(h_{3}\right)^{2} k_{1} c^{2} \omega^{3}\left(B_{33}^{0} E_{1}^{2}+B_{11}^{0} E_{3}^{2}\right)}{4\left[\omega^{2} B_{11}^{0} B_{33}^{0}-\left(h_{3} k_{1} c\right)^{2}\right]^{2}} \frac{E_{1}}{E_{3}} \\
& \mp \frac{\varepsilon_{0}\left(h_{3}\right)^{2} k_{1} c^{2} \omega^{3}\left(B_{33}^{0} E_{1}^{2}+B_{11}^{0} E_{3}^{2}\right)}{4\left[\omega^{2} B_{11}^{0} B_{33}^{0}-\left(h_{3} k_{1} c\right)^{2}\right]^{2}} \frac{\left(\left(\omega^{2} B_{11}^{0} B_{33}^{0}\right)^{2}-\left(h_{3} k_{1} c\right)^{4}\right) E_{1}^{3} E_{3}}{\left(\omega^{2} B_{11}^{0} B_{33}^{0}+\left(h_{3} k_{1} c\right)^{2}\right)^{2} E_{1}^{2} E_{3}^{2}+4\left(h_{3} k_{1} c \omega B_{11}^{0}\right)^{2} E_{3}^{4}} \\
& \mp \frac{\varepsilon_{0}\left(h_{3}\right)^{2} k_{1} c^{2} \omega^{3}\left(B_{33}^{0} E_{1}^{2}+B_{11}^{0} E_{3}^{2}\right)}{4\left[\omega^{2} B_{11}^{0} B_{33}^{0}-\left(h_{3} k_{1} c\right)^{2}\right]^{2}} \frac{2\left(\omega B_{11}^{0}\right)^{2}\left(\omega^{2} B_{11}^{0} B_{33}^{0}-\left(h_{3} k_{1} c\right)^{2}\right) E_{1} E_{3}^{3}}{\left(\omega^{2} B_{11}^{0} B_{33}^{0}+\left(h_{3} k_{1} c\right)^{2}\right)^{2} E_{1}^{2} E_{3}^{2}+4\left(h_{3} k_{1} c \omega B_{11}^{0}\right)^{2} E_{3}^{4}} .
\end{aligned}
$$

Neglecting the smallest second and third terms in the r. h. s. of Eq. (31), one can represent this equation in the following form:

$$
\begin{aligned}
& \left(S_{3}^{1}\right)_{\mathrm{Re}}=-\frac{\varepsilon_{0}\left(h_{3}\right)^{2} k_{1} c^{2} \omega^{3}\left(B_{33}^{0} E_{1}^{2}+B_{11}^{0} E_{3}^{2}\right)}{4\left[\omega^{2} B_{11}^{0} B_{33}^{0}-\left(h_{3} k_{1} c\right)^{2}\right]^{2}} \frac{E_{1}}{E_{3}} \\
& =-\frac{\varepsilon_{0}\left(h_{3}\right)^{2} k_{1} c^{2} \omega^{3}\left(B_{33}^{0} E_{1}^{2}+B_{11}^{0} E_{3}^{2}\right)}{4 \omega^{4} B_{11}^{0} B_{33}^{0}-8 \omega^{2} B_{11}^{0} B_{33}^{0}\left(h_{3} k_{1} c\right)^{2}+4\left(h_{3} k_{1} c\right)^{4}} \frac{E_{1}}{E_{3}} .
\end{aligned}
$$

When accounting that $-8 \omega^{2} B_{11}^{0} B_{33}^{0}\left(h_{3} k_{1} c\right)^{2}+4\left(h_{3} k_{1} c\right)^{4}<<4 \omega^{4}\left(B_{11}^{0} B_{33}^{0}\right)^{2}$, one gets

$$
\left(S_{3}^{1}\right)_{\mathrm{Re}} \approx-\frac{\varepsilon_{0}\left(h_{3}\right)^{2} k_{1} c^{2}\left(B_{33}^{0} E_{1}^{2}+B_{11}^{0} E_{3}^{2}\right)}{4 \omega B_{11}^{0^{2} B_{33}^{0}{ }^{2}}} \frac{E_{1}}{E_{3}}=\left(S_{1}^{1}\right)_{\operatorname{Re}} \frac{E_{1}}{E_{3}} \text {. }
$$

Finally, if a small optical anisotropy is assumed ( $B_{33}^{0} \simeq B_{11}^{0}$ ), Eq. (33) reduces to

$$
\left(S_{3}^{1}\right)_{\mathrm{Re}} \approx-\frac{1}{4} \bar{n}^{7} \varepsilon_{0}\left(h_{3}\right)^{2} c I_{\text {tot }} \frac{E_{1}}{E_{3}}=\left(S_{1}^{1}\right)_{\mathrm{Re}} \frac{E_{1}}{E_{3}},
$$

where $\bar{n}$ is the mean refractive index and $I_{t o t}$ the total light intensity propagating in crystal. As seen from Eqs. (33) and (34), the $Z$ component of the real part of the Poynting vector does not depend on the sign of vector $h_{3}$, though it is dependent on the module of the latter, as well as the total intensity of light, the refractive index, and the ratio $E_{1} / E_{3}$. Notice that the condition $E_{1} \ll<E_{3}$ gives rise to the relation $\left(S_{3}^{1}\right)_{\mathrm{Re}} \ll\left(S_{1}^{1}\right)_{\operatorname{Re}}$.

\section{Conclusions}

In the present work we have obtained a relation for the Poynting vector of electromagnetic wave that propagates in crystals possessing a so-called weak optical activity. It has been revealed that 
the Poynting vector has some transverse component that describes transverse shift of the optical beam. Such a beam drift could be explained following from the quantum properties of photon. Namely, longitudinal elliptical polarisation of electromagnetic wave caused by the weak optical activity corresponds to a transverse spin of photon. Due to spin-orbit interaction, the transverse spin results in the transverse drift of that photon.

\section{Acknowledgement}

We are thankful to Prof. O.S. Kushnir for their fruitful discussions.

\section{References}

1. Fedorov F I, 1959. On the theory of optical activity of crystals. 2. Crystals of cubic system and polar classes of medium-symmetry systems. Opt. Spektr. 6: 377-383.

2. Ivchenko E I, Permogorov S A and Sel'kin A V, 1978. Reflection of light with a change of polarization state from the real crystal boundary. Sol. St. Commun. 28: 345-348.

3. Ivchenko E L, Pevtsov A B and Sel'kin A V, 1981. Effects of optical activity on exciton luminescence of CdS. Sol. St. Commun. 39: 453-455.

4. Zil'bershtein A Kh and Solov'ev L E, 1998. Reflection of light with the change of polarization state from real crystal boundary. Opt. Spectr. 84: 549-552.

5. Lalov I J and Kojouharova N A, 2000. Reflection of electromagnetic waves at the boundary of optically active media. Bulgarian J. Phys. 27: 66-71.

6. Zapeka B, Kostyrko M and Vlokh R, 2010. On the light refraction and polarisation in the presence of weak optical activity. Ukr. J. Phys. Opt. 11: 68-73

7. Zapeka B., Kostyrko M. and Vlokh R, 2010. Coexistence of ordinary gyration and weak optical activity in crystals: eigen waves of a new type. Ukr. J. Phys. Opt. 11: 119-125.

8. Agranovich V M and Ginzburg V L, Crystal optics with spatial dispersion, and excitons Berlin, New York: Springer-Verlag (1984).

9. Agranovich V M and Ginzburg V L, 1962. Crystal optics with allowance for spatial dispersion, exciton theory. Sov. Phys.: Uspekhi. 5: 323-346.

10. Dooghin A V, Kundikova N D, Liberman V S and Zel'dovich B Ya, 1992. Optical Magnus effect. Phys. Rev. A. 45: 8204-8208.

11. Hailu Luo, Shuangchun Wen, Weixing Shu and Dianyuan Fan, 2010. Role of transverse momentum currents in optical Magnus effect in the free space. Phys. Rev. A. 81: 053826.

12. Bekshaev A, 2011. Improved theory for the polarization-dependent transverse shift of a paraxial light beam in free space. Ukr. J. Phys. Opt. 12: 10-18.

Adamenko D., Kostyrko M. and Vlokh R., 2011. On the behaviour of Poynting vector in material media with weak optical activity. Ukr.J.Phys.Opt. 12: 143-149.

Анотація. Одержано співвідношення для вектора Пойнтінта електромагнітної хвилі, яка поширюється в кристалах зі слабкою оптичною активністю. У роботі обговорено появу поперечної компоненти вектора Пойнтінта та поперечного зміщення оптичного променя внаслідок спін-орбітальної взаємодії. 